\title{
Association of endothelial activation assessed through endothelin-I precursor peptide measurement with mortality in COVID-19 patients: an observational analysis
}

Claudia Gregoriano ${ }^{1 *+} \mathbb{0}$, Dominik Damm ${ }^{1 \dagger}$, Alexander Kutz ${ }^{1}$, Daniel Koch', Selina Wolfisberg ${ }^{1}$, Sebastian Haubitz ${ }^{1,3}$, Anna Conen ${ }^{2,3}$, Luca Bernasconi ${ }^{4}$, Angelika Hammerer-Lercher ${ }^{4}$, Christoph A. Fux ${ }^{1,3}$, Beat Mueller ${ }^{1,2}$ and Philipp Schuetz ${ }^{1,2}$

\begin{abstract}
Background: Severe acute respiratory syndrome coronavirus 2 (SARS-CoV-2) disease (COVID-19) has been linked to thrombotic complications and endothelial dysfunction. We assessed the prognostic implications of endothelial activation through measurement of endothelin-I precursor peptide (proET-1), the stable precursor protein of Endothelin-1, in a well-defined cohort of patients hospitalized with COVID-19.

Methods: We measured proET-1 in 74 consecutively admitted adult patients with confirmed COVID-19 and compared its prognostic accuracy to that of patients with community-acquired pneumonia $(n=876)$ and viral bronchitis $(n=371)$ from a previous study by means of logistic regression analysis. The primary endpoint was all-cause 30-day mortality.

Results: Overall, median admission proET-1 levels were lower in COVID-19 patients compared to those with pneumonia and exacerbated bronchitis, respectively $(57.0 \mathrm{pmol} / \mathrm{l}$ vs. $113.0 \mathrm{pmol} / \mathrm{l} \mathrm{vs} .96 .0 \mathrm{pmol} / \mathrm{l}, \mathrm{p}<0.01)$. Although COVID-19 non-survivors had 1.5-fold higher admission proET-1 levels compared to survivors (81.8 pmol/I [IQR: 76 to 118] vs. 53.6 [IQR: 37 to 69]), no significant association of proET-1 levels and mortality was found in a regression model adjusted for age, gender, creatinine level, diastolic blood pressure as well as cancer and coronary artery disease (adjusted OR 0.1, 95\% Cl 0.0009 to 14.7). In patients with pneumonia (adjusted OR 25.4, 95\% Cl 5.1 to 127.4) and exacerbated bronchitis (adjusted OR 120.1, 95\% Cl 1.9 to 7499) we found significant associations of proET-1 and mortality.
\end{abstract}

Conclusions: Compared to other types of pulmonary infection, COVID-19 shows only a mild activation of the endothelium as assessed through measurement of proET-1. Therefore, the high mortality associated with COVID-19 may not be attributed to endothelial dysfunction by the surrogate marker proET-1.

Keywords: COVID-19, Endothelin-1, All-cause 30-day mortality, ProET-1, Prognostic marker, Risk assessment, SARSCoV-2

\footnotetext{
*Correspondence: c.gregoriano@gmail.com

${ }^{\dagger}$ Claudia Gregoriano and Dominik Damm contributed equally as first author

${ }^{1}$ Medical University Department of Medicine, Kantonsspital Aarau, Tellstrasse, CH-5001 Aarau, Switzerland

Full list of author information is available at the end of the article
}

\section{Background}

Coronavirus disease 2019 (COVID-19), a novel human pathogenic severe acute respiratory syndrome coronavirus counts meanwhile worldwide more than 60 million confirmed cases and more than 1.4 million deaths 
[1]. Severe acute respiratory syndrome coronavirus 2 (SARS-CoV-2) was first recognized in December 2019 in Wuhan (China) [2]. Infected patients present with a broad spectrum of symptoms causing a mild to critical disease [3]. Most of the patients (81\%) show mild infections while severe disease was reported in $14 \%$ and critical disease in 5\%, depending on the specific patient population [3]. Most frequent symptoms associated with COVID-19 are cough, fever, myalgia, headache, dyspnea, sore throat, gastrointestinal symptoms as well as loss of smell or taste [4]. Particularly, respiratory symptoms are common, because SARS-CoV-2 enters host cells via protein angiotensin-converting enzyme 2 (ACE2), which is expressed by alveolar epithelial type II cells and makes the lungs a main target for infection [5, 6]. Since many other extra-pulmonary tissues, including heart, kidney, liver and intestine, express ACE2 receptors as well, [7] symptoms associated with these organs, especially acute myocardial injury, kidney failure and diarrhea, can occur $[8,9]$.

Another target of SARS-CoV-2 infections is the endothelium, which also expresses ACE2 [10,11], leading to endothelial dysfunction as a major determinant of COVID-19. This may lead to a loss of physiological properties of the endothelium, including the ability to stimulate vasodilation, fibrinolysis, and anti-aggregation [12]. Previous research found that endothelial dysfunction plays an important role in critical illness, especially in sepsis [13].

Endothelin-1 (ET-1), mainly synthesized by activated endothelial cells which are stimulated by bacterial endotoxin and various inflammatory cytokines (TNFalpha, interleukin-6), is a potent vasoconstrictor agent [14]. Further, ET-1 is one of the major endogenous factors controlling vasotonus [15], which is responsible for blood pressure homeostasis and blood supply to individual organs $[13,16-18]$. Because of the instability of ET- 1 at room temperature and its rapid clearance from circulation, the more stable precursor fragment of ET-1, called C-terminal proendothelin-1 (proET-1), which can be measured by a sandwich immunoassay [19], is the preferable biomarker to assess endothelial dysfunction. At present, there is a lack of clinical data regarding the release of ET-1 in COVID-19 patients. As SARS-CoV-2 is targeting the endothelium, we hypothesize that circulating ET-1 levels are increased during acute illness and correlate with the risk for 30-day all-cause mortality in patients with COVID-19 requiring hospitalization. This study aimed to compare levels of proET-1 in patients with COVID-19 with those of patients with other types of respiratory infections and to investigate its association with all-cause 30-day mortality.

\section{Methods}

\section{Study design and setting}

This prospective observational study included all consecutively hospitalized adult patients ( $\geq 18$ years) with confirmed COVID-19 at the Cantonal Hospital Aarau, a tertiary care medical center in Switzerland, between February 26, 2020 and April 30, 2020.

Baseline characteristics of the analyzed COVID-19 patients have been published elsewhere [20]. In brief, COVID-19 was defined by a positive real-time reverse transcription polymerase chain reaction (RT-PCR) taken from nasopharyngeal swabs or lower respiratory tract specimens according to the WHO guidelines [21] and typical clinical symptoms of the presenting patients (e.g., respiratory symptoms with or without fever, and/or pulmonary infiltrates and/or anosmia/dysgeusia). The study was approved by the ethical committee (EKZN, 202001306) and performed in conformance with the Declaration of Helsinki ethical guidelines. All analyzed data were assessed as part of the clinical routine during the hospitalization.

\section{Data collection}

Clinical data, including socio-demographics, comorbidities as well as pre-existing medical prescription and COVID-19-specific inpatient medication were assessed until hospital discharge or death and extracted from the electronic health records. Experimental antiviral treatment was recorded if given and included hydroxychloroquine (alone or in combination with azithromycin) and sometimes tocilizumab. Comorbidities were also assessed via chart review and based on the International Statistical Classification of Diseases and Related Health Problems codes (ICD10). Additionally, patient outcomes including admission to the intensive care unit (ICU), length of hospital stay (LOS) as well as length of ICU stay were collected via chart review. 30-day mortality was collected by abstraction of hospital records, or where necessary by systematic telephone interviews. Laboratory test results were available according to clinical routine. ProET-1 was batch-tested at the end of the study and was therefore not available to the treating team during the index hospitalization.

\section{Control group}

We used patients with confirmed community-acquired pneumonia or acute and chronic exacerbated bronchitis included in a previous prospective study as a control group [22, 23]. The results of this study analyzing proET-1 have been reported in detail elsewhere [24]. In brief, from October 2006 to March 2008 consecutive patients with respiratory infection from six different hospitals located in the northern part of Switzerland were 
included and prospectively followed-up for the assessment of mortality and other endpoints.

\section{Endpoint and study objective}

The primary endpoint of this investigation was allcause 30-day mortality. For the COVID-19 patients and the control group, we assessed vital status 30 days after admission by abstraction of hospital records and/or systematic telephone interviews.

\section{Measurements of proET-1}

Plasma and serum samples on admission and during follow-up were collected in BD Vacutainer ${ }^{\circledR}$ Heparin and SST tubes. Routine left-over samples were stored at $-80{ }^{\circ} \mathrm{C}$ until analysis. Results from routine laboratory tests were recorded. C-terminal proendothelin-1 (CTproET-1) was assessed in batch using a commercially available automated fluorescent sandwich immunoassay (KRYPTOR ${ }^{\mathrm{TM}}$, B.R.A.H.M.S Thermo Fisher Scientific, Germany), as described in detail elsewhere $[25,26]$. The immunoassays have a limit of detection (LOD) of $2.94 \mathrm{pmol} / \mathrm{l}$ [27]. The functional assay sensitivity, defined as the concentration with an inter-assay coefficient of variation of $<20 \%$, is $9.78 \mathrm{pmol} / \mathrm{l}$. Values for the analytes followed a Gaussian distribution in healthy individuals without significant differences between males and females [26]. The laboratory technicians who performed the measurements were blinded to the characteristics of the patients and the characteristics of the study.

For the COVID-19 affected patients various time points during hospitalization were analyzed, depending on the available data:

- $\mathrm{T}_{0}$ (initial blood draw upon hospital admission)

- $\mathrm{T}_{1}$ (day $3 /$ day 4$)$

- $\mathrm{T}_{2}$ (day $5 /$ day 6 )

- $\mathrm{T}_{3}$ (day $7 /$ day 8 )

For the control group, blood samples for later marker measurement were collected upon admission, i.e., within the first $24 \mathrm{~h}$ upon hospital admission. ProET-1 levels were batch-measured in plasma with sandwich immunoassays (Kryptor ${ }^{\circledR}$ Thermo Scientific Biomarkers) [23].

\section{Statistical analysis}

Discrete variables are expressed as frequency (percentage) and continuous variables as medians with interquartile ranges (IQR) or mean with standard deviation (SD). In addition to descriptive statistics, we investigated the association of proET-1 levels at different time points with the primary endpoint by means of multivariable logistic regression analysis with reporting of odds ratios (OR) and corresponding 95\% confidence intervals (CI) and p-values as a measure of association. As predefined, three types of regression models were calculated, namely an unadjusted model, a model adjusted for age and gender and a model adjusted for age, gender, creatinine level, diastolic blood pressure and comorbidities. The confounders were based on previous reports published by Bhandaria et al. [28] as well as based on medical knowledge. The number of confounders was limited to avoid over-fitting of the regression models. Laboratory values with non-normal distribution were log-transformed before entering the statistical models. C-statistics was calculated as a measure of discrimination. We also validated the prognostic value of different predefined proET-1 cut-offs to predict all-cause 30-day mortality according to different proET-1 cut-offs as already presented in other non-COVID-19 studies. More specifically, one study showed the best cut-off point regarding sensitivity and specificity in predicting ICU mortality at $74 \mathrm{pmol} / \mathrm{L}$ [14]. Another study showed an optimal proET-1 cut-off of $94 \mathrm{pmol} / \mathrm{L}$ for mortality, and $154 \mathrm{pmol} / \mathrm{L}$ for prediction of bacteremia [29]. Additionally, we assessed sensitivity, specificity, positive and negative predictive values of different proET-1 cut-offs to predict 30-day mortality. Groups were compared with Wilcoxon rank sum test. A two-sided $p$-value of $<0.05$ was considered significant. Statistical analysis was performed using Stata 15.1 (StataCorp, College Station, TX, USA).

\section{Results}

Overall, we included 103 patients with confirmed COVID-19 (74 were admitted directly and 29 were transferred from other hospitals). Four patients were excluded from the analysis (decline of general consent). Further 25 cases had to be excluded because of missing aliquots for biomarker analysis $(n=9)$, or due to missing blood sampling within $24 \mathrm{~h}$ from admission $(\mathrm{n}=16)$. Thus, the final analysis encompassed 74 COVID-19 patients. In addition, 1247 control patients from a previously conducted study were used with a diagnosis of pneumonia $(n=876)$ or acute or chronic exacerbated bronchitis $(n=371)$. Figure 1 provides an overview of the study flow.

\section{Baseline characteristics}

Table 1 shows patient demographics, comorbidities as well as vital signs, laboratory findings and outcomes within 30 days in all analyzed patients and stratified by type of respiratory infection. Overall, COVID-19 patients were younger and more often male compared to the control groups. Further, patients with COVID-19 had higher initial blood pressure values with median systolic blood pressure of $141.5 \mathrm{mmHg}$ (IQR 126.0 to $156.0 \mathrm{mmHg}$ ), and diastolic blood pressure of $81.5 \mathrm{mmHg}$ (IQR 72.0 to $88.0 \mathrm{mmHg}$ ) as compared to both control groups 

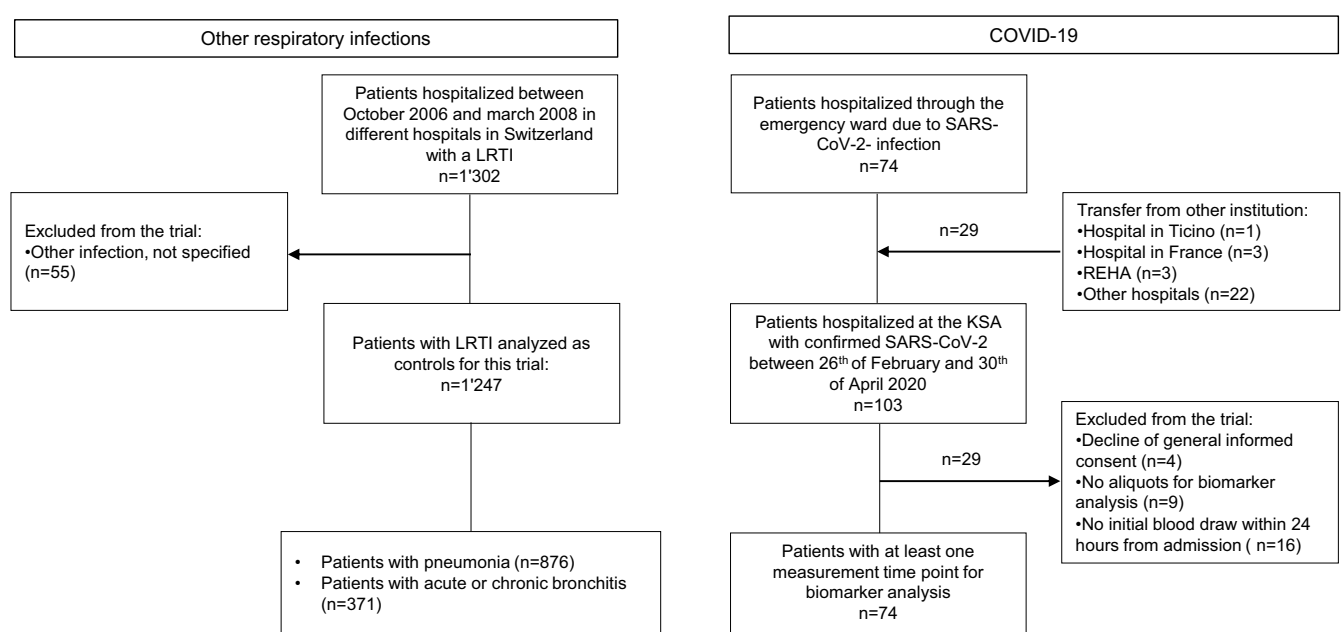

Fig. 1 Flow chart of the study. Abbreviations: KSA, cantonal hospital Aarau; LRTI, lower respiratory tract infection; SARS-CoV-2, severe acute respiratory syndrome coronavirus 2

$(\mathrm{p}<0.01)$. In general, COVID-19 patients had a higher prevalence of comorbidities. Initial laboratory findings were similar in patients with COVID-19 and controls, except for results of blood gas analysis. Patients affected by COVID-19 showed significantly lower values of $\mathrm{PCO}_{2}$ with (32 mmHg (IQR 31 to $35 \mathrm{mmHg}$ ) and without initial $\mathrm{O}_{2}$-supply ( $31 \mathrm{mmHg}$ (IQR 29 to $33 \mathrm{mmHg}$ ) at admission compared to the control patients $(\mathrm{p}<0.01)$. Regarding 30-day outcomes, COVID-19 patients had higher rates of all-cause mortality and admission to ICU. Length of stay was also longer in COVID-19 patients.

\section{Association of ProET-1 and 30-day mortality}

Median proET-1 was lower in COVID-19 patients compared to pneumonia and exacerbated bronchitis patients, respectively $(57.0 \mathrm{pmol} / \mathrm{l}$ vs. $113.0 \mathrm{pmol} / \mathrm{l}$ vs. $96.0 \mathrm{pmol} / \mathrm{l}$, $\mathrm{p}<0.01)$. Still, among all patient groups, non-survivors had higher proET-1 levels at admission compared to survivors (Table 2). In the COVID-19 population, nonsurvivors had 1.5-fold higher median admission proET-1 levels compared to survivors $(81.8 \mathrm{pmol} / \mathrm{L}$ (IQR 76 to $118 \mathrm{pmol} / \mathrm{L}$ ) vs. $53.6 \mathrm{pmol} / \mathrm{L}$ (IQR 37 to $69 \mathrm{pmol} / \mathrm{L}$ )). In patients with acute or chronic exacerbated bronchitis initial proET-1 levels were 1.8-fold higher in non-survivors when compared to patients who survived (176.5 pmol/L vs. $94 \mathrm{pmol} / \mathrm{L}$ ) and in patients with pneumonia proET-1 levels were 1.9-fold increased ( $209 \mathrm{pmol} / \mathrm{L}$ vs. $110 \mathrm{pmol} / \mathrm{L}$ ). Table 3 gives an overview of results from regression analyses and C-statistics. While proET-1 was associated with mortality in the COVID-19 population in the unadjusted analysis, this was no longer significant in the multivariable analysis adjusted for age, gender, creatinine level, diastolic blood pressure as well as cancer and coronary artery disease. A subgroup analysis regarding age, gender and comorbidities also showed no evidence for effect modification. However, for patients with pneumonia and acute or chronic exacerbated bronchitis, proET-1 was more strongly associated with mortality and associations also remained statistically significant in multivariable analyses. Regarding unadjusted discrimination for 30-day mortality, proET-1 had highest accuracy for acute or chronic exacerbated bronchitis (AUC 0.83), but was similar for pneumonia (AUC 0.75) and for COVID19 patients (AUC of 0.73).

The association of proET-1 levels at measured time points with the primary endpoint is shown in Table 4 . The highest discrimination performance of proET-1 levels was found at time point 2, i.e., day 5 or 6 of hospitalization (AUC of 0.91). A $10 \mathrm{pmol} / \mathrm{L}$ increase of proET-1 at this time point of hospitalization was associated with a $20 \%$ higher risk for 30-day mortality in the unadjusted analysis (OR $1.2(1.0$ to $1.4, \mathrm{p}=0.01)$ ). However, this was not significant in the multivariable analysis.

\section{Prognostic accuracy of proET-1 in COVID-19 at specific cut-off levels}

Prognostic accuracy of proET-1 to predict 30-day mortality was analyzed for specific proET-1 cut-offs (Table 5). Based on the Youden-Index, we found an optimal cut-off at $74 \mathrm{pmol} / \mathrm{l}$, where the sensitivity to correctly predict 30-day all-cause mortality was $76.5 \%$ (95\% CI 50.1 to 93.2) with a specificity of $77.2 \%$ (95\% CI 64.2 to $87.3 \%$ ). Furthermore, the cut-off at $74 \mathrm{pmol} / \mathrm{l}$ showed a high negative predictive value of $91.7 \%$ (95\% CI 80.0 to 97.7 ). The $94 \mathrm{pmol} / \mathrm{L}$ cut-off showed a high specificity of $91.2 \%$ (corresponding sensitivity of $29.4 \%$ ). 
Table 1 Baseline characteristics and 30-day endpoints for the analyzed study population stratified by respiratory infections

\begin{tabular}{|c|c|c|c|c|}
\hline & Pneumonia $(n=876)$ & $\begin{array}{l}\text { Acute or chronic } \\
\text { exacerbated bronchitis } \\
(n=371)\end{array}$ & SARS-CoV-2 $(n=74)$ & $p$-value \\
\hline \multicolumn{5}{|l|}{ Sociodemographics } \\
\hline Age [years] median (IQR) & $73.0(59.0,82.0)$ & $73.0(60.0,81.0)$ & $64.5(57.0,74.0)$ & $<0.01$ \\
\hline Male gender, n (\%) & $361(41.2 \%)$ & $168(45.3 \%)$ & $47(63.5 \%)$ & $<0.01$ \\
\hline \multicolumn{5}{|l|}{ Pre-existing risk-factors and medication } \\
\hline Smoker & $219(25.7 \%)$ & $123(34.1 \%)$ & $4(7.0 \%)$ & $<0.01$ \\
\hline Steroid intake & $69(8.1 \%)$ & $69(18.9 \%)$ & $1(1.4 \%)$ & $<0.01$ \\
\hline Immunosuppressive therapy & $14(1.6 \%)$ & $3(0.8 \%)$ & $3(4.1 \%)$ & 0.11 \\
\hline \multicolumn{5}{|l|}{ Comorbidities } \\
\hline Cancer, n (\%) & $110(12.6 \%)$ & $42(11.3 \%)$ & $6(8.1 \%)$ & 0.48 \\
\hline Coronary artery disease, $\mathrm{n}(\%)$ & $176(20.1 \%)$ & $85(22.9 \%)$ & $18(24.3 \%)$ & 0.42 \\
\hline Chronic heart failure, n (\%) & $151(17.2 \%)$ & $55(14.8 \%)$ & $2(2.7 \%)$ & $<0.01$ \\
\hline Solid organ transplant recipient, n (\%) & $2(0.2 \%)$ & $1(0.3 \%)$ & $0(0.0 \%)$ & 0.91 \\
\hline Chronic kidney disease, n (\%) & $196(22.4 \%)$ & $79(21.3 \%)$ & $18(24.3 \%)$ & 0.82 \\
\hline Liver cirrhosis, n (\%) & $19(2.2 \%)$ & $4(1.1 \%)$ & $0(0.0 \%)$ & 0.20 \\
\hline Obesity (BMI > 30 kg/m2), n (\%) & $35(17.7 \%)$ & $32(18.4 \%)$ & $23(31.1 \%)$ & 0.04 \\
\hline \multicolumn{5}{|l|}{ Initial vital signs } \\
\hline Blood pressure, systolic [mmHg], median (IQR) & $132.0(119.0,148.0)$ & $138.0(120.0,150.0)$ & $141.5(126.0,156.0)$ & $<0.01$ \\
\hline Blood pressure, diastolic [mmHg], median (IQR) & $74.0(65.0,82.0)$ & $78.0(66.2,85.0)$ & $81.5(72.0,88.0)$ & $<0.01$ \\
\hline Heart rate [bpm], median (IQR) & $95.0(82.0,107.0)$ & $90.0(80.0,101.0)$ & $87.5(77.5,97.5)$ & $<0.01$ \\
\hline Respiratory rate [breaths/min], median (IQR) & $20.0(16.0,25.0)$ & $20.0(16.0,26.0)$ & $22.0(18.0,27.0)$ & 0.39 \\
\hline Temperature $\left[{ }^{\circ} \mathrm{C}\right]$, median $(\mathrm{IQR})$ & $38.1(37.2,38.9)$ & $37.3(36.7,38.1)$ & $37.7(37.2,38.3)$ & $<0.01$ \\
\hline Temperature $>38^{\circ} \mathrm{C}, \mathrm{n}(\%)$ & $446(50.9 \%)$ & $105(28.3 \%)$ & $37(50.0 \%)$ & $<0.01$ \\
\hline $\mathrm{SpO}_{2}[\%]$, median (IQR) & $93.0(89.0,96.0)$ & $93.0(89.0,96.0)$ & $93.0(87.8,95.3)$ & 0.99 \\
\hline \multicolumn{5}{|l|}{ Initial laboratory findings } \\
\hline Blood gas analysis for ambient air, $\mathrm{n}(\%)$ & $n=455$ & $n=198$ & $n=54$ & \\
\hline $\mathrm{FiO}_{2}[\%]$, median (IQR) & 21 & 21 & 21 & N/A \\
\hline $\mathrm{PO}_{2}[\mathrm{mmHg}]$, median (IQR) & $62(57,76)$ & $66(58,76)$ & $68(61,72)$ & 0.9 \\
\hline $\mathrm{PCO}_{2}[\mathrm{mmHg}]$, median (IQR) & $35(31,38)$ & $36(32,41)$ & $31(29,33)$ & $<0.01$ \\
\hline Lactate $[\mathrm{mmol} / \mathrm{I}]$, median (IQR) & $1.6(1.0,2.3)$ & $1.4(1.1,1.6)$ & $1.3(0.9,1.7)$ & 0.1 \\
\hline Blood gas analysis for initial $\mathrm{O}_{2}, \mathrm{n}(\%)$ & $n=421$ & $n=173$ & $\mathrm{n}=20$ & \\
\hline FiO2 [\%], median (IQR) & $32(28,36)$ & $28(28,36)$ & $47(32,95)$ & $<0.01$ \\
\hline $\mathrm{PO}_{2}[\mathrm{mmHg}]$, median (IQR) & $60(53,70)$ & $62(53,74)$ & $65(56,75)$ & 0.24 \\
\hline $\mathrm{PCO}_{2}[\mathrm{mmHg}]$, median (IQR) & $35(31,39)$ & $38(34,47)$ & $32(31,35)$ & $<0.01$ \\
\hline Lactate $[\mathrm{mmol} / \mathrm{l}]$, median (IQR) & $1.3(1.0,1.8)$ & $1.0(0.9,1.4)$ & $1.2(1.0,1.5)$ & 0.11 \\
\hline Leukocytes [G/L], median (IQR) & $12.0(9.0,16.4)$ & $9.7(7.5,13.3)$ & $7.3(4.6,8.7)$ & $<0.01$ \\
\hline Sodium [mmol/L], median (IQR) & $136.0(133.0,138.0)$ & $136.0(134.0,139.0)$ & $136.0(133.0,139.0)$ & $<0.01$ \\
\hline Glucose $[\mathrm{mmol} / \mathrm{L}]$, median (IQR) & $7.1(6.0,8.5)$ & $6.7(5.8,7.9)$ & $6.7(5.9,8.6)$ & 0.01 \\
\hline Urea [mmol/L], median (IQR) & $7.1(4.9,10.5)$ & $6.5(4.4,9.6)$ & $5.9(4.4,9.4)$ & 0.06 \\
\hline CRP [mg/L], median (IQR) & $154.5(74.2,251.9)$ & $41.0(14.0,98.4)$ & $94.3(49.9,150.0)$ & $<0.01$ \\
\hline PCT [ $\mu \mathrm{g} / \mathrm{L}]$, median (IQR) & $0.46(0.15,2.66)$ & $0.12(0.08,0.20)$ & $0.11(0.05,0.26)$ & $<0.01$ \\
\hline Creatinine $[\mu \mathrm{mol} / \mathrm{L}]$, median (IQR) & $89.0(69.0,113.0)$ & $84.0(66.0,106.0)$ & $91.0(77.0,115.0)$ & 0.02 \\
\hline Pro-Endothelin-1 [pmol/L], median (IQR) & $113(81.0,169.0)$ & $96.0(67.0,139.0)$ & $57.0(41.4,81.8)$ & $<0.01$ \\
\hline \multicolumn{5}{|l|}{ Outcomes within 30 days } \\
\hline ICU care, n (\%) & $80(9.1 \%)$ & $13(3.5 \%)$ & $22(29.7 \%)$ & $<0.01$ \\
\hline 30-day mortality, n (\%) & $49(5.6 \%)$ & $10(2.7 \%)$ & $17(23.0 \%)$ & $<0.01$ \\
\hline Length of stay [day], median (IQR) & $8.0(5.0,13.0)$ & $7.0(3.0,11.0)$ & $9.0(5.0,14.0)$ & $<0.01$ \\
\hline
\end{tabular}


Table 1 (continued)

$\mathrm{BMI}$, Body-Mass-Index; bpm, beats per minute; C, Celsius; $\mathrm{CRP}, \mathrm{C}$-reactive protein; $\mathrm{FiO}_{2}$, fraction of inspired oxygen; ICU, intensive care unit; IQR, interquartile range; $\mathrm{mmHg}$, millimeter of mercury; $\mathrm{NA}$, not applicable; $\mathrm{pCO}_{2}$, partial pressure of carbon dioxide; $\mathrm{PCT}$, procalcitonin; $\mathrm{PO}_{2}$, partial pressure of oxygen; $\mathrm{SpO} \mathrm{O}_{2}$, oxygen saturatio

Table 2 ProET-1 values and different cut-offs stratified by the analyzed respiratory infections and by survivors and non-survivors

\begin{tabular}{|c|c|c|c|c|c|c|}
\hline & \multicolumn{2}{|l|}{ Pneumonia $(n=876)$} & \multicolumn{2}{|c|}{$\begin{array}{l}\text { Acute or chronic exacerbated bronchitis } \\
(\mathrm{n}=371)\end{array}$} & \multicolumn{2}{|l|}{ SARS-CoV-2 $(n=74)$} \\
\hline & Survivors $(n=827)$ & $\begin{array}{l}\text { Non-Survivors } \\
n=49 \text { ) }\end{array}$ & Survivors $(n=361)$ & $\begin{array}{l}\text { Non-Survivors } \\
(n=10)\end{array}$ & Survivors $(n=57)$ & $\begin{array}{l}\text { Non-Survivors } \\
(n=17)\end{array}$ \\
\hline \multicolumn{7}{|l|}{ proET-1 values } \\
\hline $\begin{array}{l}\text { proET-1 overall } \\
\text { [pmol/L], median } \\
\text { (IQR) }\end{array}$ & $110.0(80.0,163.0)$ & $209.0(135.0,328.0)$ & $94.0(67.0,133.0)$ & $176.5(155.0,230.0)$ & $53.6(37.0,69.0)$ & $81.8(76.0,118.0)$ \\
\hline \multicolumn{7}{|l|}{ proET-1 cut-offs, n (\%) } \\
\hline$<74[\mathrm{pmol} / \mathrm{L}]$ & $162(19.6 \%)$ & $3(6 \%)$ & $114(31.6 \%)$ & $0(0 \%)$ & $44(77.2 \%)$ & $4(24 \%)$ \\
\hline$\geq 74[\mathrm{pmol} / \mathrm{L}]$ & $141(17.0 \%)$ & $4(8 \%)$ & $68(18.8 \%)$ & $1(10 \%)$ & $8(14.0 \%)$ & $8(47 \%)$ \\
\hline$>94[\mathrm{pmol} / \mathrm{L}]$ & $292(35.3 \%)$ & $9(18 \%)$ & 115 (31.9\%) & $1(10 \%)$ & $3(5.3 \%)$ & $3(18 \%)$ \\
\hline$>154[\mathrm{pmol} / \mathrm{L}]$ & $232(28.1 \%)$ & $33(67 \%)$ & $64(17.7 \%)$ & $8(80 \%)$ & $2(3.5 \%)$ & $2(12 \%)$ \\
\hline \multicolumn{7}{|c|}{ proET-1 median-cut-off, n, (\%) } \\
\hline$<107[\mathrm{pmol} / \mathrm{L}]$ & $378(45.7 \%)$ & $9(18 \%)$ & $215(59.6 \%)$ & $1(10 \%)$ & $52(91.2 \%)$ & $12(71 \%)$ \\
\hline$\geq 107[\mathrm{pmol} / \mathrm{L}]$ & $449(54.3 \%)$ & $40(82 \%)$ & $146(40.4 \%)$ & $9(90 \%)$ & $5(8.8 \%)$ & $5(29 \%)$ \\
\hline
\end{tabular}

IQR, interquartile range; proET-1, proEndothelin-1; SARS-CoV-2, severe acute respiratory syndrome coronavirus 2

Table 3 Logistic regression analyses forAssociation of initial proET-1 values and 30-day mortality in the different respiratory infections

\begin{tabular}{|c|c|c|c|}
\hline & Pneumonia ( $n=876$ ) & $\begin{array}{l}\text { Acute or chronic exacerbated } \\
\text { bronchitis }(n=371)\end{array}$ & SARS-CoV-2 $(n=74)$ \\
\hline \multicolumn{4}{|c|}{ Regression analysis, $\mathrm{OR}^{\mathrm{C}}(95 \% \mathrm{Cl}$ ), p-value } \\
\hline Unadjusted model & 51.1 (15.0 to 174.2), $p<0.01$ & 215.3 (10.9 to 4246.2), $p<0.01$ & $32.2(2.3$ to 455.5$), p=0.01$ \\
\hline Multivariable model $1^{\text {a }}$ & 36.7 (9.7 to 138.4), $p<0.01$ & 82.0 (2.8 to 2394.0$), p=0.01$ & $6.6(0.2$ to 193.3$), p=0.27$ \\
\hline Multivariable model $2^{\mathrm{b}}$ & $25.4(5.1$ to 127.4$), p<0.01$ & $120.7(1.9$ to 7499.7$), p=0.02$ & 0.1 (0.0009 to 14.7$), p=0.38$ \\
\hline \multicolumn{4}{|l|}{ Discrimination statistics AUC (95\% Cl) } \\
\hline AUC (95\% Cl) Unadjusted model & 0.75 (0.67 to 0.83$)$ & 0.85 (0.75 to 0.95$)$ & 0.73 (0.58 to 0.89$)$ \\
\hline
\end{tabular}

AUC, area under the curve; $\mathrm{Cl}$, confidence interval; OR, odd ratio; SARS-CoV-2, severe acute respiratory syndrome coronavirus 2;

${ }^{\text {a }}$ Adjusted for age and gender

${ }^{b}$ Adjusted for age, gender, creatinine level, diastolic blood pressure as well as cancer and coronary artery disease

' Odds ratio for $1 \mathrm{pmol} / \mathrm{L}$ increase of proET-1

\section{Discussion}

This prospective study evaluated endothelial activation through measurement of initial proET-1 in patients with confirmed COVID-19, as well as patients with other types of respiratory infections. Although we expected an increase in proET-1 in COVID-19 patients compared to other types of respiratory infections, our results showed quite the opposite. In fact, our results indicate a less pronounced activation of proET-1 in COVID-19 compared to other respiratory tract infections with non-significant associations with mortality in multivariable analyses. Although proET-1 provided some prognostic information regarding mortality, discrimination analysis based on C-statistic showed only a moderate prognostic accuracy. These results suggest that in COVID-19 patients proET-1 is not a powerful marker for calculating morbidity and mortality in contrast to its validity in other respiratory infections.

Several pro-inflammatory cytokines and prognostic markers from different pathways have been investigated in patients with COVID-19 [30,31]. Yet, to our knowledge, there is a lack of data looking at endothelial biomarkers including proET-1 in patients affected by COVID-19.

There is a strong rationale for looking at proET-1 in COVID-19 patients based on results of previous research 
Table 4 Logistic regression analyses for different proET-1 cut-offs at different time points. Crude and adjusted association of different proET-1 cut-offs at different time points and 30-day mortality

\begin{tabular}{|c|c|c|c|c|c|c|c|}
\hline & Survivors $(n=57)$ & $\begin{array}{l}\text { Non-Survivors } \\
(n=17)\end{array}$ & p-value & AUC & $\begin{array}{l}\text { Univariable OR } \\
(95 \% \mathrm{Cl}), \mathrm{p} \text {-value }\end{array}$ & $\begin{array}{l}\text { Multivariabl }{ }^{\mathrm{a}} \mathrm{OR}^{\mathrm{c}} \\
(95 \% \mathrm{Cl}), \mathrm{p} \text {-value }\end{array}$ & $\begin{array}{l}\text { Multivariable }{ }^{b} \text { OR }^{c} \\
(95 \% \mathrm{Cl}), \mathrm{p} \text {-value }\end{array}$ \\
\hline \multicolumn{8}{|c|}{ proET-1 Time point 0 (within $24 \mathrm{~h}$ form admission), $\mathrm{n}=74$} \\
\hline $\begin{array}{l}\text { proET-1 overall, } \\
\text { median (IQR) }\end{array}$ & $53.6(37.0,69.0)$ & $81.8(76.0,118.0)$ & $<0.01$ & 0.74 & $\begin{array}{l}1.2(1.1 \text { to } 1.4) \\
\mathrm{p}<\mathbf{0 . 0 1}\end{array}$ & $\begin{array}{c}1.1(0.9 \text { to } 1.4) \\
p=0.12\end{array}$ & $\begin{array}{l}0.9(0.6 \text { to } 1.2) \\
p=0.35\end{array}$ \\
\hline \multicolumn{8}{|c|}{ proET-1- 74.0-cut-off [pmol/L], n (\%) } \\
\hline$\leq 74.0$ & $44(77 \%)$ & $4(24 \%)$ & $<0.01$ & & Reference & Reference & Reference \\
\hline$>74.0$ & $13(23 \%)$ & $13(76 \%)$ & & & $\begin{array}{l}11.0(3.1 \text { to } 39.6) \\
p<0.01\end{array}$ & $\begin{array}{l}4.8(1.0 \text { to } 22.0) \\
p=0.05\end{array}$ & $\begin{array}{l}1.9(0.3 \text { to } 13.0) \\
p=0.50\end{array}$ \\
\hline \multicolumn{8}{|c|}{ proET-1- 94.0-cut-off [pmol/L], n (\%) } \\
\hline$\leq 94.0$ & $52(91 \%)$ & $12(71 \%)$ & 0.03 & & Reference & Reference & Reference \\
\hline$>94.0$ & $5(9 \%)$ & $5(29 \%)$ & & & $\begin{array}{l}4.3(1.1 \text { to } 17.4) \\
p=0.04\end{array}$ & $\begin{array}{l}2.6(0.5 \text { to } 14.0) \\
p=0.27\end{array}$ & $\begin{array}{l}1.0(0.1 \text { to } 14.0) \\
p=0.99\end{array}$ \\
\hline \multicolumn{8}{|c|}{ proET-1- 154.0-cut-off [pmol/L], n (\%) } \\
\hline$\leq 154.0$ & $55(96 \%)$ & $15(88 \%)$ & 0.19 & & Reference & Reference & NA \\
\hline$>154.0$ & $2(4 \%)$ & $2(12 \%)$ & & & $\begin{array}{l}3.7(0.5 \text { to } 28.2) \\
p=0.2\end{array}$ & $\begin{array}{l}3.3(0.3 \text { to } 38.2) \\
p=0.34\end{array}$ & NA \\
\hline \multicolumn{8}{|c|}{ proET-1- median-cut-off [pmol/L], n (\%) } \\
\hline$\leq 106$ & $52(91 \%)$ & $12(71 \%)$ & 0.03 & & Reference & Reference & Reference \\
\hline$>106$ & $5(9 \%)$ & $5(29 \%)$ & & & $\begin{array}{l}4.3(1.1 \text { to } 17.4) \\
p=\mathbf{0 . 0 4}\end{array}$ & $\begin{array}{l}2.6(0.5 \text { to } 14.0) \\
p=0.27\end{array}$ & $\begin{array}{l}1.0(0.07 \text { to } 14.0) \\
p=0.99\end{array}$ \\
\hline \multicolumn{8}{|c|}{ proET-1 Time point 1 (day 3/day 4 of hospitalization), $n=55$} \\
\hline $\begin{array}{l}\text { proET-1 overall, } \\
\text { median (IQR) }\end{array}$ & $56.9(44.5,77.3)$ & $124.3(95.4,156.0)$ & $<0.01$ & 0.81 & $\begin{array}{l}1.2(1.0 \text { to } 1.04) \\
p<0.01\end{array}$ & $\begin{array}{l}1.2(1.0 \text { to } 1.4) \\
p=\mathbf{0 . 0 3}\end{array}$ & $\begin{array}{l}1.3(1.0 \text { to } 1.8) \\
p=0.76\end{array}$ \\
\hline \multicolumn{8}{|c|}{ proET-1- 74.0-cut-off [pmol/L], n (\%) } \\
\hline$\leq 74.0$ & $30(70 \%)$ & $2(17 \%)$ & $<0.01$ & & Reference & Reference & Reference \\
\hline$>74.0$ & $13(30 \%)$ & $10(83 \%)$ & & & $\begin{array}{l}11.5(2.2 \text { to } 60.2) \\
p<0.01\end{array}$ & $\begin{array}{l}8.7(1.5 \text { to } 52.3) \\
p=\mathbf{0 . 0 2}\end{array}$ & $\begin{array}{l}72.0(1.0 \text { to } 5257.0) \\
p=\mathbf{0 . 0 5}\end{array}$ \\
\hline \multicolumn{8}{|c|}{ proET-1- 94.0-cut-off [pmol/L], n (\%) } \\
\hline$\leq 94.0$ & $37(86 \%)$ & $3(25 \%)$ & $<0.01$ & & Reference & Reference & Reference \\
\hline$>94.0$ & $6(14 \%)$ & $9(75 \%)$ & & & $\begin{array}{l}18.5(3.9 \text { to } 88.5) \\
\mathrm{p}<\mathbf{0 . 0 1}\end{array}$ & $\begin{array}{l}12.4(2.2 \text { to } 68.3) \\
p<\mathbf{0 . 0 1}\end{array}$ & $\begin{array}{l}78.6(1.0 \text { to } 5688.0) \\
p=\mathbf{0 . 0 5}\end{array}$ \\
\hline \multicolumn{8}{|c|}{ proET-1- 154.0-cut-off [pmol/L], n (\%) } \\
\hline$\leq 154.0$ & $41(95 \%)$ & $9(75 \%)$ & 0.03 & & Reference & Reference & Reference \\
\hline$>154.0$ & $2(5 \%)$ & $3(25 \%)$ & & & $\begin{array}{l}6.8(0.9 \text { to } 47.0) \\
p=0.05\end{array}$ & $\begin{array}{l}6.2(0.7 \text { to } 54.3) \\
p=0.10\end{array}$ & $\begin{array}{l}68.0(0.2 \text { to } 18,750.0), \\
p=0.14\end{array}$ \\
\hline \multicolumn{8}{|c|}{ proET-1- median-cut-off [pmol/L], n (\%) } \\
\hline$\leq 63.4$ & $27(63 \%)$ & $1(8 \%)$ & $<0.01$ & & Reference & Reference & NA \\
\hline$>63.4$ & $16(37 \%)$ & $11(92 \%)$ & & & $\begin{array}{l}18.6(2.2 \text { to } 157.5) \\
p<\mathbf{0 . 0 1}\end{array}$ & $\begin{array}{l}17.0(1.8 \text { to } 160.7) \\
p=\mathbf{0 . 0 1}\end{array}$ & NA \\
\hline \multicolumn{8}{|c|}{ proET-1 Time point 2 (day 5/day 6 of hospitalization), $n=45$} \\
\hline $\begin{array}{l}\text { proET-1 overall, } \\
\text { median (IQR) }\end{array}$ & $54.0(39.0,91.2)$ & $163.2(107.2,217.6)$ & $<0.01$ & 0.91 & $\begin{array}{l}1.2(1.0 \text { to } 1.4) \\
p=\mathbf{0 . 0 1}\end{array}$ & $\begin{array}{l}1.1(1.0 \text { to } 1.3) \\
p=\mathbf{0 . 0 3}\end{array}$ & N/A \\
\hline \multicolumn{8}{|c|}{ proET-1- 74.0-cut-off [pmol/L], n (\%) } \\
\hline$\leq 74.0$ & $26(72 \%)$ & $1(11 \%)$ & $<0.01$ & & Reference & Reference & NA \\
\hline$>74.0$ & $10(28 \%)$ & $8(89 \%)$ & & & $\begin{array}{l}20.8(2.3 \text { to } 188.3) \\
p<\mathbf{0 . 0 1}\end{array}$ & $\begin{array}{l}19.3(1.6 \text { to } 238.0) \\
p=\mathbf{0 . 0 2}\end{array}$ & NA \\
\hline \multicolumn{8}{|c|}{ proET-1- 94.0-cut-off [pmol/L], n (\%) } \\
\hline$\leq 94.0$ & $28(78 \%)$ & $1(11 \%)$ & $<0.01$ & & Reference & Reference & NA \\
\hline$>94.0$ & $8(22 \%)$ & $8(89 \%)$ & & & $\begin{array}{l}28.0(3.0 \text { to } 258.4) \\
p<0.01\end{array}$ & $\begin{array}{l}25.2(2.01 \text { to } 307.2) \\
\quad p=\mathbf{0 . 0 1}\end{array}$ & NA \\
\hline \multicolumn{8}{|c|}{ proET-1 - 154.0-cut-off [pmol/L], n (\%) } \\
\hline$\leq 154.0$ & $33(92 \%)$ & $4(44 \%)$ & $<0.01$ & & Reference & Reference & Reference \\
\hline
\end{tabular}


Table 4 (continued)

\begin{tabular}{|c|c|c|c|c|c|c|c|}
\hline & Survivors $(n=57)$ & $\begin{array}{l}\text { Non-Survivors } \\
(n=17)\end{array}$ & p-value & AUC & $\begin{array}{l}\text { Univariable OR } \\
(95 \% \mathrm{Cl}), \mathrm{p} \text {-value }\end{array}$ & $\begin{array}{l}\text { Multivariabl' }{ }^{a} R^{c} \\
(95 \% \mathrm{Cl}), \mathrm{p} \text {-value }\end{array}$ & $\begin{array}{l}\text { Multivariable }{ }^{b} \text { OR }^{c} \\
(95 \% \mathrm{Cl}), \mathrm{p} \text {-value }\end{array}$ \\
\hline$>154.0$ & $3(8 \%)$ & $5(56 \%)$ & & & $\begin{array}{l}13.8(2.3 \text { to } 80.6) \\
p<\mathbf{0 . 0 1}\end{array}$ & $\begin{array}{l}9.8(1.4 \text { to } 70.3) \\
p=\mathbf{0 . 0 2}\end{array}$ & $\begin{array}{l}15.6(0.5 \text { to } 499.2) \\
p=0.12\end{array}$ \\
\hline \multicolumn{8}{|c|}{ proET-1- median-cut-off [pmol/L], n (\%) } \\
\hline$\leq 59.5$ & $23(64 \%)$ & $0(0 \%)$ & $<0.01$ & & NA & NA & NA \\
\hline$>59.5$ & $13(36 \%)$ & $9(100 \%)$ & & & NA & NA & NA \\
\hline \multicolumn{8}{|c|}{ proET-1 Time point 3 (day $7 /$ day 8 of hospitalization), $n=31$} \\
\hline $\begin{array}{l}\text { proET-1 overall, } \\
\text { median (IQR) }\end{array}$ & $56.5(38.6,101.7)$ & $108.6(97.8,169.4)$ & 0.04 & 0.8 & $\begin{array}{c}1.1(0.9 \text { to } 1.3) \\
p=0.06\end{array}$ & $\begin{array}{c}1.1(1.0 \text { to } 1.3) \\
p=0.12\end{array}$ & $\begin{array}{c}1.2(0.9 \text { to } 1.5) \\
p=0.16\end{array}$ \\
\hline \multicolumn{8}{|c|}{ proET-1- 74.0-cut-off [pmol/L], n (\%) } \\
\hline$\leq 74.0$ & $15(58 \%)$ & $0(0 \%)$ & 0.02 & & NA & NA & NA \\
\hline$>74.0$ & $11(42 \%)$ & $5(100 \%)$ & & & NA & NA & NA \\
\hline \multicolumn{8}{|c|}{ proET-1- 94.0-cut-off [pmol/L], n (\%) } \\
\hline$\leq 94.0$ & $17(65 \%)$ & $1(20 \%)$ & 0.06 & & Reference & Reference & Reference \\
\hline$>94.0$ & $9(35 \%)$ & $4(80 \%)$ & & & $\begin{array}{c}7.6(0.7 \text { to } 78.1) \\
p=0.09\end{array}$ & $\begin{array}{c}4.6(0.4 \text { to } 54.7) \\
p=0.23\end{array}$ & $\begin{array}{l}11.3(0.2 \text { to } 593.4) \\
p=0.23\end{array}$ \\
\hline \multicolumn{8}{|c|}{ proET-1- 154.0-cut-off [pmol/L], n (\%) } \\
\hline$\leq 154.0$ & $23(88 \%)$ & $3(60 \%)$ & 0.11 & & Reference & Reference & Reference \\
\hline$>154.0$ & $3(12 \%)$ & $2(40 \%)$ & & & $\begin{array}{c}5.1(0.6 \text { to } 44.1) \\
p=0.14\end{array}$ & $\begin{array}{c}2.6(0.2 \text { to } 26.7) \\
p=0.43\end{array}$ & $\begin{array}{c}3.6(0.1 \text { to } 86.5) \\
p=0.43\end{array}$ \\
\hline \multicolumn{8}{|c|}{ proET-1- median-cut-off [pmol/L], n (\%) } \\
\hline$\leq 81.65$ & $16(62 \%)$ & $0(0 \%)$ & 0.01 & & NA & NA & NA \\
\hline$>81.65$ & $10(38 \%)$ & $5(100 \%)$ & & & NA & NA & NA \\
\hline
\end{tabular}

Bold values indicate statistical significant

AUC, area under the curve; $\mathrm{Cl}$, confidence interval; NA, not applicable; OR, odd ratio; proET-1, proEndothelin-1

a Adjusted for age and gender

${ }^{b}$ Adjusted for age, gender, creatinine level, diastolic blood pressure as well as cancer and coronary artery disease

' Odds ratio for $10 \mathrm{pmol} / \mathrm{L}$ increase of proET-1

Table 5 Prognostic accuracy of different proET-1 cut-offs at baseline for patients with COVID-19

\begin{tabular}{|c|c|c|c|c|}
\hline $\begin{array}{l}\text { ProET-1 Cut-off value } \\
\text { [pmol/I] }\end{array}$ & Sensitivity $(95 \% \mathrm{Cl})$ & Specificity $(95 \% \mathrm{Cl})$ & $\begin{array}{l}\text { Positive predictive value } \\
(95 \% \mathrm{Cl})\end{array}$ & $\begin{array}{l}\text { Negative } \\
\text { predictive value } \\
(95 \% \mathrm{Cl})\end{array}$ \\
\hline $74.0 \mathrm{pmol} / \mathrm{l}$ & 76.5 (50.1 to 93.2) & $77.2(64.2$ to 87.3$)$ & 50.029 .9 to 70.1$)$ & 91.7 (80.0 to 97.7) \\
\hline $94.0 \mathrm{pmol} / \mathrm{l}$ & 29.4 (10.3 to 56.0) & $91.2(80.7$ to 97.1$)$ & 50.0 (18.7 to 81.3) & 81.3 (69.5 to 89.9) \\
\hline $154.0 \mathrm{pmol} / \mathrm{l}$ & $11.8(1.5$ to 36.4$)$ & 96.5 (87.9 to 99.6) & 50.0 (6.8 to 93.2$)$ & $78.6(67.1$ to 87.5$)$ \\
\hline \multicolumn{5}{|l|}{ ProET-1 median pmol/l] } \\
\hline $107.0 \mathrm{pmol} / \mathrm{l}$ & 29.4 (10.3 to 56.0) & $91.2(80.7$ to 97.1$)$ & $50.0(18.7$ to 81.3$)$ & 81.369 .5 to 89.9$)$ \\
\hline
\end{tabular}

$\mathrm{Cl}$, confidence interval; proET-1, proEndothelin-1

in other types of infections. More specifically, the prognostic relevance of proET-1 was analyzed in several prior studies focusing on critical-ill patients with and without sepsis [14], patients with septic shock [32], patients with myocardial injury and myocardial dysfunction due to septic shock [33], as well as patients with community acquired pneumonia [29]. For critically ill patients, results showed elevated proET-1 as an independent risk factor for ICU admission and overall mortality regardless of sepsis diagnosis [14]. Another study found an about eightfold increase of proET-1 in the plasma of septic patients with increasing severity of infection [32] and showed that proET-1 correlates with disease severity. Further, it can be used as independent predictor for mortality in patients with community-acquired pneumonia. However, our results suggest that proET-1 may not be as important in COVID-19 compared to other infections. In fact, our results showed that patients with COVID19 had lower initial proET-1 levels, when compared to patients with pneumonia and exacerbated bronchitis. 
Clinically, COVID-19 initially has less effect on the (cardio) vascular system but is more affecting the lungs. COVID-19 patients rarely need vasopressor support despite high severity of illness, while in sepsis hypotension and shock is a hallmark of the disease. This may be explained by the fact that COVID-19 is a systemic disease that affects many organs, especially different organs and tissues, where the ACE2 receptors are expressed allowing the virus to enter the cells. Some authors report that the density of ACE2 in each tissue may correlate with the severity of COVID-19 in that tissue [7, 34-38]. However, other than in sepsis, patients with COVID-19 remain hemodynamically stable and therefore, proET-1 and ET-1 remain low. Another reason might be, that SARS-CoV-2 enters endothelial cells via ACE2 receptors and leads to cell damage that stops them from releasing proET-1, as already described in a similar way for SARS-CoV when entering pancreatic islet cells and suppressing or destroying them [39].

In our study population, COVID-19 patients were mainly transferred to ICU due to the need for non-invasive and/or invasive ventilation support. In contrast, reason for ICU admission in patients with other respiratory infection is often due to shock and need for vasopressor support. Other than in septic patients, where the need of pressure support and rising levels of the vasoconstrictor ET-1 are crucial, the reason for severe disease and death in patients with COVID-19 may rather be related to the infection-mediated endothelial injury and endothelialitis. This in turn may trigger excessive thrombin production, inhibit fibrinolysis, and activate complement pathways, which consequently initiate thrombo-inflammation and can lead to micro-thromboembolism and microvascular dysfunctions [40].

Compared to prior studies [14], the optimal cut-off for the prediction of 30-day mortality found in our study was lower with a value of $74 \mathrm{pmol} / \mathrm{L}$. This cut-off showed the best sensitivity and specificity to predict all-cause 30-day mortality in patients with COVID-19. The use of higher cut-offs like in prior studies [29], showed only a very low sensitivity.

\section{Limitations}

This study has several limitations. The results of this analysis have to be interpreted in the context of the study design: First, due to the single center design of this study the number of analyzed COVID-19 cases was small and external validation is needed. Second, clinical data were limited and not all evaluated laboratory parameters and characteristics were available for all patients, resulting in few missing data. Third, due to incomplete data, left atrial size, which represent a known confounder of proET-1 levels, was not considered in the adjusted regression models.

\section{Conclusion}

In conclusion, as compared to other types of pulmonary infection, COVID-19 causes increases of proET-1 concentrations to a lesser extent, which might be explained by either only a mild activation of the endothelium or by the reduced possibility to produce the hormone from damaged endothelium. Thus, we could not find any evidence that the high mortality associated with COVID19 can be estimated by the endothelial function marker proET-1. Based on our results, the use of proET-1 for prognostic risk stratification in patients with COVID-19 is not recommended.

\section{Abbreviations}

ACE2: Angiotensin-converting enzyme 2; BMI: Body mass index; bpm: beats per minute; C: Celsius; Cl: Confidence intervals; COVID-19: Coronavirus disease 2019; CRP: C-reactive protein; ET-1: Endothelin-1; ICD-10: International statistical classification of diseases and related health problems codes; ICU: Intensive care unit; IQR: Interquartile ranges; LOD: Limit of detection; LOS: Length of hospital stay; LRTI: Lower respiratory tract infection; NS: Non-survivors; proET1: ProEndothelin-1; OR: Odds ratios; RAAS: Renin-angiotensin-aldosterone system; ROC-AUC: Area under the operating receiver curve; RT-PCR: Real-time reverse transcription polymerase chain reaction; S: Survivors; SARS-CoV-2: Severe acute respiratory syndrome coronavirus 2; SD: Standard deviation; TNFalpha: Tumor necrosis factor-alpha; WHO: World health organization.

\section{Acknowledgements}

We thank all participating patients and their families, and all healthcare workers at the Cantonal Hospital Aarau for their great dedication to reduce the burden of this severe disease.

\section{Authors' contributions}

CG, AK, and PS had the idea, wrote the protocol and initiated the study. CG managed the trial and CG, DK, SH collected data. LB and AHL managed the laboratory investigations. CG performed the statistical analyses and CG, DD and PS drafted the manuscript. AK, SW, DK, SH, AC, LB, AHL, KS, CAF and BM, amended and commented on the manuscript. All authors read and approved the final manuscript.

\section{Funding}

This study was funded by the Research Council KSA (Kantonsspital Aarau). Costs for biomarker measurement (reagents) were sponsored by ThermoFisher Scientific, BRAHMS, Henningsdorf (Germany). The funding agency have no bearing on the study design, data collection and analysis or writing of the manuscript.

Availability of data and materials

The datasets used and analyzed during the current study are available from the corresponding author on reasonable request.

\section{Declarations}

Ethics approval and consent to participate

This study was approved by the local ethics Committee northwest/central Switzerland (EKZN, 2020-01306). Written general informed consent was provided from all analyzed patients.

Consent for publication

Not applicable. 


\section{Competing interests}

PS and BM received research support paid to the Institution from Thermofisher, bioMerieux, Roche Diagnostics, Nestle Health Science and Abbott Nutrition. KS reports grants from Thermofisher and Pfizer. All other authors reported no conflicts of interest.

\section{Author details}

${ }^{1}$ Medical University Department of Medicine, Kantonsspital Aarau, Tellstrasse, CH-5001 Aarau, Switzerland. ${ }^{2}$ University of Basel, Basel, Switzerland. ${ }^{3}$ Department of Infectious Diseases and Hospital Hygiene, Kantonsspital Aarau, Switzerland. ${ }^{4}$ Institute of Laboratory Medicine, Kantonsspital Aarau, Aarau, Switzerland.

Received: 15 February 2021 Accepted: 7 May 2021 Published online: 13 May 2021

\section{References}

1. World Health Organization (WHO). Coronavirus disease (COVID-19) Weekly Epidemiological Update. https://www.who.int/publications/m/ item/weekly-epidemiological-update---27-october-2020. 2020

2. (WHO). WHO. Coronavirus disease 2019 (COVID-19) - Weekly Epidemiological Update. https://www.who.int/docs/default-source/coronaviru se/situation-reports/20200824-weekly-epi-update.pdf?sfvrsn $=80698$ 6d1_4.

3. Wu Z, McGoogan JM. Characteristics of and important lessons from the coronavirus disease 2019 (COVID-19) outbreak in China: summary of a report of 72314 cases from the Chinese center for disease control and prevention. JAMA. 2020;323:1239.

4. Stokes EK, Zambrano LD, Anderson KN, Marder EP, Raz KM, El Burai FS, et al. Coronavirus disease 2019 case surveillance-United States, January 22-May 30, 2020. MMWR Morb Mortal Wkly Rep. 2020;69(24):759-65.

5. Zhang H, Penninger JM, Li Y, Zhong N, Slutsky AS. Angiotensinconverting enzyme 2 (ACE2) as a SARS-CoV-2 receptor: molecular mechanisms and potential therapeutic target. Intensive Care Med. 2020;46(4):586-90.

6. Zhou P, Yang XL, Wang XG, Hu B, Zhang L, Zhang W, et al. A pneumonia outbreak associated with a new coronavirus of probable bat origin. Nature. 2020;579(7798):270-3.

7. Gheblawi M, Wang K, Viveiros A, Nguyen Q, Zhong JC, Turner AJ, et al. Angiotensin-converting enzyme 2: SARS-CoV-2 receptor and regulator of the renin-angiotensin system: celebrating the 20th anniversary of the discovery of ACE2. Circ Res. 2020;126(10):1456-74.

8. Durvasula R, Wellington T, McNamara E, Watnick S. COVID-19 and kidney failure in the acute care setting: our experience from seattle. Am J Kidney Dis. 2020;76(1):4-6.

9. Ronco C, Reis T. Kidney involvement in COVID-19 and rationale for extracorporeal therapies. Nat Rev Nephrol. 2020;16(6):308-10.

10. Lovren F, Pan Y, Quan A, Teoh H, Wang G, Shukla PC, et al. Angiotensin converting enzyme-2 confers endothelial protection and attenuates atherosclerosis. Am J Physiol Heart Circ Physiol. 2008;295(4):H1377-84.

11. Sluimer JC, Gasc JM, Hamming I, van Goor H, Michaud A, van den Akker LH, et al. Angiotensin-converting enzyme 2 (ACE2) expression and activity in human carotid atherosclerotic lesions. J Pathol. 2008;215(3):273-9.

12. Godo S, Shimokawa H. Endothelial functions. Arterioscler Thromb Vasc Biol. 2017;37(9):e108-14.

13. Aird WC. The role of the endothelium in severe sepsis and multiple organ dysfunction syndrome. Blood. 2003;101(10):3765-77.

14. Buendgens L, Yagmur E, Bruensing J, Herbers U, Baeck C, Trautwein C, et al. C-terminal proendothelin-1 (CT-proET-1) is associated with organ failure and predicts mortality in critically ill patients. J Intensive Care. 2017:5:25.

15. Barton M, Yanagisawa M. Endothelin: 20 years from discovery to therapy. Can J Physiol Pharmacol. 2008:86(8):485-98.

16. Lee WL, Slutsky AS. Sepsis and endothelial permeability. N Engl J Med 2010;363(7):689-91.

17. Shah R. Endothelins in health and disease. Eur J Intern Med. 2007:18(4):272-82
18. Wanecek M, Weitzberg E, Rudehill A, Oldner A. The endothelin system in septic and endotoxin shock. Eur J Pharmacol. 2000;407(1-2):1-15.

19. Papassotiriou J, Morgenthaler NG, Struck J, Alonso C, Bergmann A. Immunoluminometric assay for measurement of the C-terminal endothelin-1 precursor fragment in human plasma. Clin Chem. 2006;52(6):1144-51.

20. Gregoriano C, Koch D, Haubitz S, Conen A, Fux CA, Mueller B, et al. Characteristics, predictors and outcomes among 99 patients hospitalised with COVID-19 in a tertiary care centre in Switzerland: an observational analysis. Swiss Med Wkly. 2020;150:w20316.

21. WHO. Clinical management of severe acute respiratory infection when novel conovirus (nCov) infection is supsected; interim guidance, 25 January 2020. Published January 25, 2020.

22. Schuetz $P$, Christ-Crain M, Thomann R, Falconnier C, Wolbers M, Widmer I, et al. Effect of procalcitonin-based guidelines vs standard guidelines on antibiotic use in lower respiratory tract infections: the ProHOSP randomized controlled trial. JAMA. 2009;302(10):1059-66.

23. Kutz A, Grolimund E, Christ-Crain M, Thomann R, Falconnier C, Hoess $C$, et al. Pre-analytic factors and initial biomarker levels in communityacquired pneumonia patients. BMC Anesthesiol. 2014;14:102.

24. Schuetz P, Christ-Crain M, Wolbers M, Schild U, Thomann R, Falconnier $C$, et al. Procalcitonin guided antibiotic therapy and hospitalization in patients with lower respiratory tract infections: a prospective, multicenter, randomized controlled trial. BMC Health Serv Res. 2007;7:102.

25. Christ-Crain M, Morgenthaler NG, Struck J, Harbarth S, Bergmann A, Muller B. Mid-regional pro-adrenomedullin as a prognostic marker in sepsis: an observational study. Crit Care. 2005;9(6):R816-24.

26. Morgenthaler NG, Struck J, Alonso C, Bergmann A. Measurement of midregional proadrenomedullin in plasma with an immunoluminometric assay. Clin Chem. 2005;51(10):1823-9.

27. Hausfater P, Claessens YE, Martinage A, Joly LM, Lardeur JY, Der Sahakian G, et al. Prognostic value of PCT, copeptin, MR-proADM, MRproANP and CT-proET-1 for severe acute dyspnea in the emergency department: the BIODINER study. Biomarkers. 2017;22(1):28-34.

28. Bhandari SS, Davies JE, Struck J, Ng LL. Plasma C-terminal proEndothelin-1 (CTproET-1) is affected by age, renal function, left atrial size and diastolic blood pressure in healthy subjects. Peptides. 2014;52:53-7.

29. Schuetz P, Stolz D, Mueller B, Morgenthaler NG, Struck J, Mueller C, et al. Endothelin-1 precursor peptides correlate with severity of disease and outcome in patients with community acquired pneumonia. BMC Infect Dis. 2008;8:22

30. Han H, Ma Q, Li C, Liu R, Zhao L, Wang W, et al. Profiling serum cytokines in COVID-19 patients reveals IL-6 and IL-10 are disease severity predictors. Emerg Microbes Infect. 2020;9(1):1123-30.

31. Del Valle DM, Kim-Schulze S, Hsin-Hui H, Beckmann ND, Nirenberg S, Wang B, et al. An inflammatory cytokine signature helps predict COVID-19 severity and death. medRxiv. 2020.

32. Schuetz P, Christ-Crain M, Morgenthaler NG, Struck J, Bergmann A, Muller B. Circulating precursor levels of endothelin-1 and adrenomedullin, two endothelium-derived, counteracting substances, in sepsis. Endothelium. 2007;14(6):345-51.

33. Lundberg OH, Bergenzaun L, Ryden J, Rosenqvist M, Melander O, Chew MS. Adrenomedullin and endothelin-1 are associated with myocardial injury and death in septic shock patients. Crit Care. 2016;20(1):178.

34. Gallagher TM, Buchmeier MJ. Coronavirus spike proteins in viral entry and pathogenesis. Virology. 2001;279(2):371-4.

35. Jia HP, Look DC, Shi L, Hickey M, Pewe L, Netland J, et al. ACE2 receptor expression and severe acute respiratory syndrome coronavirus infection depend on differentiation of human airway epithelia. J Virol. 2005:79(23):14614-21.

36. Li M, Chen L, Zhang J, Xiong C, Li X. The SARS-CoV-2 receptor ACE2 expression of maternal-fetal interface and fetal organs by single-cell transcriptome study. PLoS One. 2020;15(4):e0230295.

37. Perico L, Benigni A, Remuzzi G. Should COVID-19 concern nephrologists? Why and to what extent? THE emerging impasse of angiotensin blockade. Nephron. 2020;144(5):213-21.

38. Xu H, Zhong L, Deng J, Peng J, Dan H, Zeng $X$, et al. High expression of ACE2 receptor of 2019-nCoV on the epithelial cells of oral mucosa. Int J Oral Sci. 2020;12(1):8. 
39. Yang JK, Lin SS, Ji XJ, Guo LM. Binding of SARS coronavirus to its receptor damages islets and causes acute diabetes. Acta Diabetol. 2010:47(3):193-9.

40. Varga Z, Flammer AJ, Steiger P, Haberecker M, Andermatt R, Zinkernagel AS, et al. Endothelial cell infection and endotheliitis in COVID-19. Lancet. 2020;395(10234):1417-8.

\section{Publisher's Note}

Springer Nature remains neutral with regard to jurisdictional claims in published maps and institutional affiliations.
Ready to submit your research? Choose BMC and benefit from:

- fast, convenient online submission

- thorough peer review by experienced researchers in your field

- rapid publication on acceptance

- support for research data, including large and complex data types

- gold Open Access which fosters wider collaboration and increased citations

- maximum visibility for your research: over 100M website views per year

At BMC, research is always in progress.

Learn more biomedcentral.com/submissions 